\title{
Prussian blue nanoparticles and their analogues for application to cancer theranostics
}

\section{"..Prussian blue nanoparticles and their analogues could be useful for potential cancer theranostics in the near future."}

First draft submitted: 15 December 2015; Accepted for publication: 22 January 2016;

Keywords: biocompatible $\bullet$ cancer theranostics $\bullet$ drug delivery $\bullet$ nanomedicine $\bullet$ Prussian blue nanoparticles $\bullet$ US FDA-approved drug

\section{Prussian blue nanoparticles: background \& applications}

Prussian blue (PB), a mixed valance hexacyanomatelate salt, was historically known as ancient dark blue pigment. The discovery and 290 years of history of PB has been well documented by Kraft and Berlin [1]. PB (ferric hexacyanoferrate) and its analogues are well known inorganic salts of co-ordination complexes that show interesting electro, photochemical, biochemical and magnetic properties [2]. The porous nature and special unique properties of $\mathrm{PB}$ make them suitable candidates for several biological, medicinal and other applications including photonics, magneto-optical devices, catalysis, energy storage, staining agent for histology study, chelating agent for the removal of heavy metals and radioactive elements [3-5]. Among all applications, Prussian blue nanoparticles (PBNPs) have been extensively used for several biomedical applications including drug delivery due to several advantages like their biocompatible nature, biodegradability, ease to synthesize, controllable shape and morphology, and low production cost. Additionally, Prussian blue capsule, also known as Radiogardase (an active pharmaceutical ingredient), has been approved by the US FDA in 2003 as a safe oral antidote and effective for the treatment of internal contamination with radioactive and nonradioactive metals (thallium, cesium). It works by the combination of thallium and cesium in the intestine and the combined complex is removed from the body through stool.

\section{Theranostic applications of Prussian} blue nanoparticles \& their analogues Over the past 10 years, nanomedicine has become an emerging field of nanotechnology applications to health. Recently, several groups including ours have investigated the development of metal nanoparticle-based theranostic approaches for the treatment of several diseases including cancer, antibacterial activity and cardiovascular related diseases [5-11]. Hence, design, development, fabrication metal nanoparticles or inorganic complex nanoparticles, especially PBNPs, through a simple, efficient and economically cheap method is a very important area for nanobiotechnology research because of its medicinal applications [9,12-14]. In this context, we demonstrated a simple, efficient, fast, template free, cost-effective and ecofriendly method for the synthesis of copper analogues of Prussian blue (Cu-PBNPs) nanoparticles that are stable and biocompatible, observed by several analytical techniques and in vitro assays. The $\mathrm{Cu}-\mathrm{PBNPs}$ was synthesized by the interaction of $\mathrm{CuSO}_{4}$ solution and $\mathrm{K}_{3} \mathrm{Fe}(\mathrm{CN})_{6}$ at room temperature with different volume ratio. The PBNPs with $1: 1$ molar ratio of $\mathrm{CuSO}_{4}$ and $\mathrm{K}_{3} \mathrm{Fe}(\mathrm{CN})_{6}$ is considered as optimized PBNPs based on the

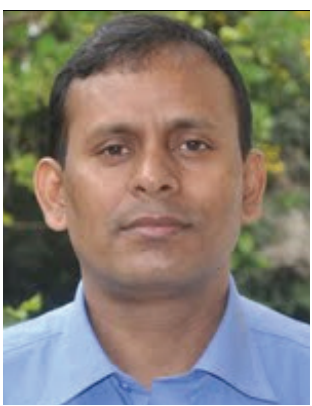

Chitta Ranjan Patra Biomaterials Group, CSIR-Indian Institute of Chemical Technology, Uppal Road, Tarnaka, Hyderabad 500007, Telangana, India and

Academy of Scientific \& Innovative Research (AcSIR), Taramani,

Chennai- 600113, India Tel.: +914027191480 Fax: +914027160387 crpatra@iict.res.in
Future
Medicine fsg 
stability, biocompatibility and release kinetics of the drug release. These nanoparticles successfully deliver the doxorubicin drug to cancer cells. Additionally, these nanoparticles exhibit the selective quenching of fluorescence of doxorubicin compared with PB analogues with other metals ions. Considering its multifunctional biological activities including sustained and slow release of anticancer drug (doxorubicin: DOX), delivery of DOX to the cancer cells, nuclear targeting property and selective fluorescence quenching property to metal ions, these nanoparticles could be useful for potential theranostics applications in biomedical sciences [5].

Since pediatric brain tumor (PBT) is a primary cause of death in children, the development of molecularly-specific imaging agents is urgently needed to monitor disease progression and therapeutic treatment. Dumont et al., demonstrated the manganese-containing Prussian blue nanoparticles (Mn-PBNPs) (where $\mathrm{Mn}^{2+}$ ions are used for paramagnetic property) as molecular MRI and fluorescence-based imaging agent for the diagnostic treatment of PBT [15]. The functionalization of manganese-containing nanoparticles consists of fluorescent avidin that works for dual action (enabling fluorescence imaging help in the attachment for biotinylated ligands that target PBT) based on the orthotopic mouse model of PBTs. Jing et al. demonstrated the photoabsorbing agent of core/shell nanoparticles of gold and Prussian blue (Au@PBNPs) for photothermal therapy and photoacoustic (PA) imaging because of high molar extinction coefficient in near- infrared (NIR) region and high photostability [16]. These nanoparticles could be used as an effective cancer diagnostic with noninvasive therapy for cancer therapy in future. Wang et al. demonstrated that PBNPs could be useful for the delivery of DNA drugs towards cancer cells after surface functionalization with 11-mercaptoundecanoic acid (MUA) and conjugation of drug, decoy oligodeoxynucleotide (dODN) [17]. The drug delivery system (dODN@ MUA-PBNPs) was internalized inside the cytoplasmic compartment of the cells after endocytosis and able to kill cancer cells. Noroozifar $e t$ al. reported the synthesis of silver hexacyanoferrate nanoparticles that could be useful for the simultaneous determination of ascorbic acid, dopamine and uric acid present in biological fluid (urine and human blood serum) samples with good sensitivity and selectivity [18]. Dumont et al. reported the synthesis, characterization, stability, cytotoxicity, fluorescence and MR imaging capabilities of the biofunctionalized Prussian blue NPs that could be used as multimodal molecular imaging agents in near future [19]. Another study reported by Fu et al. demonstrated the fabrication of a new core shell theranostic agent $\left(\mathrm{Fe}_{3} \mathrm{O}_{4} @ \mathrm{~PB}\right.$ NPs) by the combination of two FDA approved materials (PBNPs and superparamagnetic $\mathrm{Fe}_{3} \mathrm{O}_{4} \mathrm{NP}$ ). Here PBNPs are used as NIR-driven photothermal agent for photothermal therapy treatment of cancer due to its strong optical absorbance in NIR region and high photothermal conversion efficiency and has been used as the contrast enhancement agent for MRI. The newly designed nanoshell will have great potential as a new theranostic nanomedicine for clinical applications [13]. Fiorito et al. demonstrated the synthesis of PBNPs using $\mathrm{FeCl}_{3}$ and $\mathrm{K}_{3} \mathrm{Fe}(\mathrm{CN})_{6}$ and showed the immobilized NPs in multilayer structure for the development of potential amperometric transducer for oxidase-enzyme-based biosensors. The technique could be useful as a potential tool for a biosensing device [12]. Lian et al. designed single crystalline highly biocompatible coordination polymer PBNPs and successfully delivered the cis-plation to the bladder cancer cells (T24 cells). The PBNPs are nontoxic because cyanide groups are tightly bound to the Fe atom. The authors claim hollow PBNPs could be useful as an efficient drug delivery in cancer therapy [14]. Biocompatible Prussian blue nanoparticles that can penetrate the cell membrane have also been investigated for dual action including as an MRI agent as well as for a therapeutic purpose due to the presence of super paramagnetic iron oxides (imaging as well as drug delivery vehicle) [7]. Stable and narrow-sized (around $4 \mathrm{~nm}$ ) PBNPs protected by polyvinylpyrrolidone have been utilized as a sensitive label-free immunodetection (detection limit from $200 \mu \mathrm{g} \mathrm{ml}^{-1}$ to $34 \mathrm{pg}$ human IgG ml${ }^{-1}$ ) for a cancer (parathyroid hormone-related protein, $\mathrm{PTHrP}$ ), observed by in vitro experiments [6]. Jing et al. demonstrated the fabrication of a multifunctional nanoagent system after modification of hollow Prussian blue nanoparticles (HPBNPs: as photothermal agent and drug carrier,) using hyaluronic acid grafting polyethylene glycol (HA-g-PEG: capping agent to increase the colloidal stability, prolonged blood circulation time, targeting ability because of cancer targeting HA to the surface of HeLa cells over-expressing the CD44 receptor) and 10-hydroxycamptothecin (as chemotherapeutic agent) for tumor-targeted thermochemotherapy. The synergetic effect (combination of both photothermal therapy and chemotherapy) of multifunctional HPBNPs in both in vitro and in vivo systems shows enhanced therapeutic efficacy compared with either photothermal therapy or chemotherapy alone. Therefore, the multifunctional HPBNPs could be useful as promising theranostic nanomedicine (noninvasive imaging and remote-controlled therapy) in the near future [9]. Li et al. reported the development of a strategy for sensitive electrochemical immunoassay of human tissue polypeptide antigen, cancer marker using 
Prussian blue nanoparticles-doped nanogold microsphere (AuPB) as promoter [20]. The electrochemical immunoassay shows nice electrochemical responses for the detection of tissue polypeptide antigen at low concentration range $\left(5 \mathrm{pg} \mathrm{ml}^{-1}\right)$. The authors validated the data for the analysis of eight clinic human serum specimens and eight spiked serum samples and the results corroborate with the referenced enzyme-linked immunosorbent assay (ELISA) method.

\section{Potential long-term toxicity issues \& future perspective}

Extensive safety assessment of any nanoparticles for investigating toxicity must be evaluated in a preclinical model to satisfy regulators as well as patients before administration in the human body or clinical trial. Hence, efficacy, biosafety, metabolic long-term fate (in vitro and in vivo), pharmacokinetics profiles, potential long-term toxicity study of PBNPs in a mouse model should be systematically evaluated before using these novel materials in a clinical trial [11]. Several reports support the biocompatible nature and nontoxicity of PBNPs, both in in vitro and in vivo systems, may be due to cyano groups being tightly bound to the iron metal in PBNPs. It was reported that the citratecoated PBNPs exhibited no significant cytotoxicity [7]. The nontoxic Prussian blue has been extensively used

\section{References}

1 Kraft A, Berlin GG. On the discovery and history of prussian blue. Bull. Hist. Chem. 33(2), 61-67 (2008).

2 Johansson A, Widenkvist E, Lu J, Boman M, Jansson U. Fabrication of high-aspect-ratio Prussian blue nanotubes using a porous alumina template. Nano Lett. 5(8), 1603-1606 (2005).

3 Roy X, Hui JK, Rabnawaz M, Liu G, MacLachlan MJ. Prussian blue nanocontainers: selectively permeable hollow metal-organic capsules from block ionomer emulsion-induced assembly. J. Am. Chem.Soc. 133(22), 8420-8423 (2011).

4 Hu M, Furukawa S, Ohtani R et al. Synthesis of Prussian blue nanoparticles with a hollow interior by controlled chemical etching. Angew. Chem. Int. Ed. Engl. 51(4), 984-988 (2012).

5 Mukherjee S, Rao BR, Sreedhar B, Paik P, Patra CR. Copper Prussian blue analogue: investigation into multifunctional activities for biomedical applications. Chem. Commun. (Camb.) 51(34), 7325-7328 (2015).

6 Espinoza-Castaneda M, de la Escosura-Muniz A, Chamorro A, de Torres C, Merkoci A. Nanochannel array device operating through Prussian blue nanoparticles for sensitive label-free immunodetection of a cancer biomarker. Biosens. Bioelectron. 67, 107-114 (2015).

7 Shokouhimehr M, Soehnlen ES, Hao JH et al. Dual purpose Prussian blue nanoparticles for cellular imaging and drug as a pigment in industry and for artists since 1704 . Nowadays, PB is used in several applications including catalysis, energy storage, chelating agent and medicinal and biological applications. Recently, Prussian blue capsule (Radiogardase) has been approved by FDA as an oral antidote for the elimination of radioactive and nonradioactive cesium and thallium poisoning metal ions from human body. Since PBNP is a nontoxic and FDA-approved compound and already used clinically as an antidote for the elimination of radioactive and nonradioactive thallium and cesium poisoning metal ions from the human body, therefore we strongly believe that Prussian blue nanoparticles and their analogues could be useful for potential cancer theranostics in the near future.

\section{Financial \& competing interests disclosure}

This research was supported by the 'Ramanujan Fellowship grant' (SR/S2/RJN-04/2010; GAP0305), DST-New Delhi and 'CSIR 12 ${ }^{\text {th }}$ Five Year Plan (FYP) project (ADD: CSC0302) to CR Patra. The author has no other relevant affiliations or financial involvement with any organization or entity with a financial interest in or financial conflict with the subject matter or materials discussed in the manuscript apart from those disclosed.

No writing assistance was utilized in the production of this manuscript.

delivery: a new generation of T-1-weighted MRI contrast and small molecule delivery agents. J. Mater. Chem. 20, 5251-5259 (2010).

8 Mukherjee S, Chowdhury D, Kotcherlakota R et al. Potential theranostics application of bio-synthesized silver nanoparticles (4-in-1 system). Theranostics 4(3), 316-335 (2014).

9 Jing LJ, Shao SM, Wang Y, Yang YB, Yue XL, Dai ZF. Hyaluronic acid modified hollow prussian blue nanoparticles loading 10-hydroxycamptothecin for targeting thermochemotherapy of cancer. Theranostics 6(1), 40-53 (2015).

10 Patra CR, Kim JH, Pramanik K et al. Reactive oxygen species drivenangiogenesis by inorganic nanorods. Nano Lett. 11(11), 4932-4938 (2011).

11 Patra CR. Graphene oxides and the angiogenic process. Nanomedicine (Lond.) 10(19), 2959-2962 (2015).

12 Fiorito PA, Goncales VR, Ponzio EA, de Torresi SI. Synthesis, characterization and immobilization of Prussian blue nanoparticles. A potential tool for biosensing devices. Chem. Commun. (Camb.) (3), 366-368 (2005).

13 Fu G, Liu W, Li Y et al. Magnetic Prussian blue nanoparticles for targeted photothermal therapy under magnetic resonance imaging guidance. Bioconjug. Chem. 25(9), 1655-1663 (2014).

14 Lian HY, Hu M, Liu CH, Yamauchi Y, Wu KCW. Highly biocompatible, hollow coordination polymer nanoparticles 
as cisplatin carriers for efficient intracellular drug delivery. Chem. Commun. 48, 5151-5153 (2012).

15 Dumont MF, Yadavilli S, Sze RW, Nazarian J, Fernandes R. Manganese-containing Prussian blue nanoparticles for imaging of pediatric brain tumors. Int. J. Nanomedicine 9, 2581-2595 (2014).

16 Jing L, Liang X, Deng Z et al. Prussian blue coated gold nanoparticles for simultaneous photoacoustic/CT bimodal imaging and photothermal ablation of cancer. Biomaterials 35, 5814-5821 (2014).

17 Wang SJ, Chen CS, Chen LC. Prussian blue nanoparticles as nanocargoes for delivering DNA drugs to cancer cells. Sci. Technol. Adv. Mat. 14 (4), 044405 (2013).
18 Noroozifar M, Khorasani-Motlagh M, Taheri A. Preparation of silver hexacyanoferrate nanoparticles and its application for the simultaneous determination of ascorbic acid, dopamine and uric acid. Talanta 80, 1657-1664 (2010).

19 Dumont MF, Hoffman HA, Yoon PR et al. Biofunctionalized gadolinium-containing prussian blue nanoparticles as multimodal molecular imaging agents. Bioconjugate Chem. 25, 129-137 (2014).

20 Li QF, Lou FM, Tang DP. Biofunctional nanogold microsphere doped with Prussian blue nanoparticles for sensitive electrochemical immunoassay of cancer marker. Anal. Methods-Uk. 6, 3442-3448 (2014). 